\title{
Preservation of calcium pyrophosphate dihydrate crystals: effect of Mayer's haematoxylin staining period
}

T Ohira, K Ishikawa

\begin{abstract}
Objective-To clarify the deleterious effects of Mayer's haematoxylin staining procedure which result in a decrease in, or complete loss of, the number of calcium pyrophosphate dihydrate (CPPD) crystals, and to determine the proper staining period for preserving the crystals in a histological paraffin section of articular tissues.

Methods-Paraffin sections of CPPD crystal-bearing articular tissues of six patients were stained with Mayer's haematoxylin for 3, 8, or 15 minutes, and subsequently with eosin for one minute. The specimens were examined with an Olympus BHS polarised light microscope. The pH of Mayer's haematoxylin solution was measured with a TOA pH meter.

Results-Positive birefringent CPPD crystals were seen clearly in all specimens stained with Mayer's haematoxylin for three minutes. The specimens stained for eight minutes showed a reduced number of crystals. No crystals were seen in the specimens stained for 15 minutes. Ordinary light microscopy showed no notable differences in the stainability of nucleus, cell membrane, and their surrounding tissues among specimens when stained with Mayer's haematoxylin for either 3, 8, or 15 minutes. The pH of Mayer's haematoxylin solution was 2.31 .

Conclusions-To find CPPD crystals in the paraffin sections of articular tissues, the staining period with Mayer's haematoxylin should be limited to three minutes. The longer the staining period, the greater the reduction in the number of crystals owing to the strong acidity of the haematoxylin solution. A staining period of 15 minutes causes a complete loss of CPPD crystals.

(Ann Rheum Dis 2001;60:80-82)
\end{abstract}

Hospit, Japan

T Ohira

Ishikawa Orthopaedic and Rheumatism

Hospital, Kumamoto, Japan

K Ishikawa

Correspondence to: Dr T Ohira, Ohira

Orthopaedic Hospital, 15-30

Miyako-machi Hyuga-shi,

Miyazaki, 883-0043, Japan

gonhyuga@mnet.ne.jp

Accepted for publication 1 May 2000 are not seen in a synovial fluid, a histological examination of crystals in articular tissues becomes crucial. As a routine procedure, histological specimens are treated with haematoxylin and eosin $(\mathrm{H} \& \mathrm{E}){ }^{2}$ The staining periods of haematoxylin-namely, from five minutes to 15 minutes $^{23}$ or more, ${ }^{2}$ are often determined by the investigator's personal preference. Histological specimens of articular tissues bearing CPPD crystals, treated with $\mathrm{H} \& \mathrm{E}$, occasionally show no light microscopic evidence of crystals. ${ }^{45}$ This false negative result seems to be caused by decalcification of calciumcontaining CPPD crystals through the strong acidity of the haematoxylin solution. ${ }^{6}$

This study was carried out to clarify the effects of Mayer's haematoxylin staining procedure causing a loss of CPPD crystals and to determine the proper staining period for preserving the crystals in histological specimens.

\section{Methods}

TISSUE SAMPLES

The articular cartilage, meniscus, and synovial membrane obtained during total knee arthroplasty of six patients (two male and four female, mean (SD) age 72.1 (3.5) years), who fulfilled the criteria of Ryan and McCarty for definite CPPD crystal deposition disease, ${ }^{1}$ were examined. The identification of CPPD crystals in the articular tissues of three patients was performed by $x$ ray diffraction according to the method described earlier by Ohira and Ishikawa. $^{7}$

\section{TISSUE PREPARATIONS}

The articular tissues were fixed in $10 \%$ neutral buffered formalin, embedded in paraffin, and cut into $6 \mu \mathrm{m}$ slices. The deparaffinised sections were stained with Mayer's haematoxylin for either 15,8 , or 3 minutes and, subsequently, with eosin for one minute. Staining was performed according to the method described by Luna. ${ }^{2}$ Haematoxylin powders (Merk, Darmstadt, Germany), 1 g, were dissolved in $100 \mathrm{ml}$ of distilled water and $50 \mathrm{~g}$ aluminium potassium sulphate. $12 \mathrm{H}_{2} \mathrm{O}$, (Katayama Chemical, Osaka, Japan) was dissolved in $800 \mathrm{ml}$ of distilled water. These two solutions were mixed and $0.2 \mathrm{~g}$ sodium iodate, $1 \mathrm{~g}$ citric acid, and $50 \mathrm{~g}$ chloral hydrate were added to the solution, and shaken until all the components were completely dissolved. Eosin solution was produced by adding $1 \mathrm{~g}$ eosin $\mathrm{Y}$ (Merk, Darmstadt, Germany) to $100 \mathrm{ml}$ distilled water. Acetic acid, $4 \mathrm{ml}$, and $800 \mathrm{ml}$ $95 \%$ ethyl alcohol were added to $100 \mathrm{ml}$ of the eosin solution, when used.
MEASUREMENT OF PH

The $\mathrm{pH}$ of Mayer's haematoxylin solution at room temperature was measured with a TOA pH meter HM-30S (TOA Electronics, Tokyo, Japan). 
LIGHT AND COMPENSATED POLARISED LIGHT MICROSCOPIC OBSERVATIONS

Mayer's haematoxylin and eosin stained preparations were observed by an ordinary light microscope and under compensated polarised light by the use of a first order red compensator with an Olympus BHS microscope.

\section{Results}

The $\mathrm{pH}$ of the final solution of Mayer's haematoxylin at room temperature was 2.31 .

Under polarised light, the preparations of all patients stained with Mayer's haematoxylin for three minutes clearly showed weakly positive birefringent CPPD crystals within oval or round shaped deposits (fig $1 \mathrm{~A}$ ). The number of crystals was markedly reduced in the preparations treated with Mayer's haematoxylin for eight minutes and no crystals were seen in the preparations stained for 15 minutes (fig 1B). Under ordinary light, the stainability of the nucleus was slightly decreased in the preparations stained with Mayer's haematoxylin for
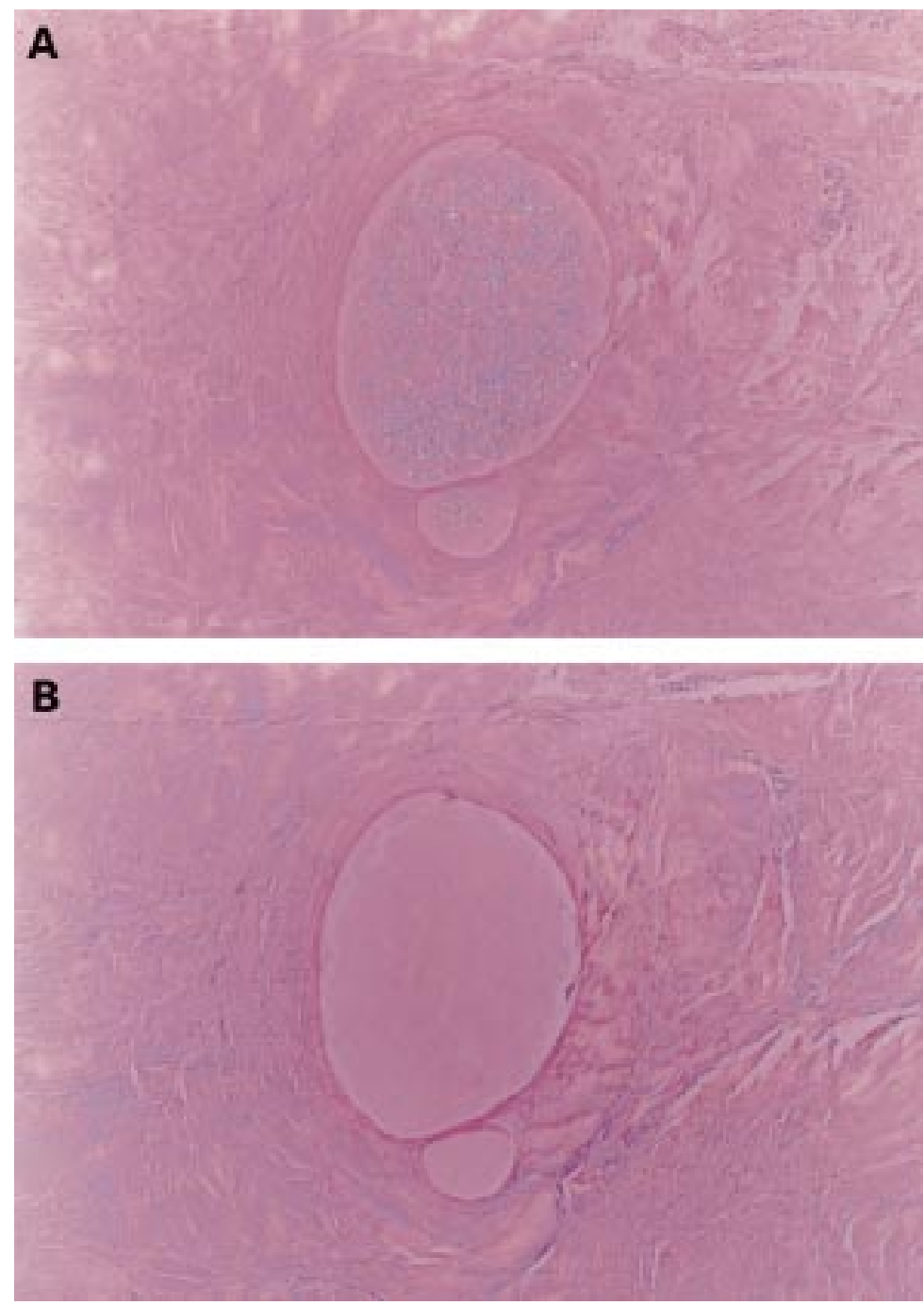

Figure 1 (A) Photomicrograph showing abundant calcium pyrophosphate dihydrate crystals with a weakly positive birefringence in the paraffin section of meniscus treated with Mayer's haematoxylin for three minutes and with eosin for one minute. (Under compensated polarised light, original magnification $\times 100$ ). (B) No calcium pyrophosphate dihydrate crystals are seen in the paraffin section of the meniscus shown in fig $1 \mathrm{~A}$ that was treated with Mayer's haematoxylin for 15 minutes and with eosin for one minute. (Under compensated polarised light, original magnification $\times 100$ ). three minutes but no other notable differences in the stainability were noted in the cytoplasm and its surrounding tissue among the specimens stained with Mayer's haematoxylin for 3, 8 or 15 minutes, respectively.

\section{Discussion}

A histological study of 79 elderly patients (mean age 67) who underwent a total knee or hip arthroplasty for severe osteoarthritic joint destruction showed a high rate $(36 / 79(46 \%))$ of CPPD crystal deposition in the articular tissues obtained at surgery. ${ }^{8}$ Most of the specimens obtained from the 36 patients with CPPD crystal deposition showed necrotic bone fragments, infiltration of multinucleated phagocytes, fibrinoid degeneration, and/or lymphoid follicle formation in the synovial membrane, and marked destructive changes of subchondral bone, including massive bone necrosis, fragmentation, and infiltration of multinucleated osteoclasts. In contrast, these histological changes were scarcely seen in the specimens obtained from the 43 patients with no deposition of CPPD crystals. $^{8}$

The high rate of deposition of CPPD crystals in destructive osteoarthropathy and the fact that CPPD crystal deposition disease is common in the elderly, ${ }^{9}$ but is occasionally overlooked, ${ }^{10}$ seem to indicate that the number of patients with destructive osteoarthropathy of CPPD crystal deposition disease ${ }^{1}$ is probably greater than generally considered. Several pitfalls in making a correct diagnosis may lead to an unawareness of CPPD crystal deposition disease. These include: (a) no radiological evidence of articular chondrocalcinosis ${ }^{10-12}$ in patients with small amounts of crystal deposits or with severe osteoarthropathy accompanying an attrition, abrasion, or loss of articular tissues bearing CPPD crystals; (b) difficulty in observing positive birefringent CPPD crystals in synovial fluid ${ }^{12-14}$ or in specimens treated with $\mathrm{H} \& \mathrm{E}^{4}{ }^{5}$

The results of our study showed that a prolonged period of staining with Mayer's haematoxylin, such as 15 minutes, caused a complete loss of CPPD crystals. We speculate that the CPPD crystals might have dissolved through decalcification or fallen off the slide through a disruption of the adjacent matrix owing to the strong acidity ( $\mathrm{pH} 2.31$ ) of Mayer's haematoxylin solution.

Fortunately, a staining period of three minutes preserved a large number of CPPD crystals showing a positive birefringence without sacrificing the excellent stainability of haematoxylin. We hope that the results of this study will be referred to in Mayer's H\&E staining procedures of histological specimens and thus contribute to reaching a correct diagnosis of CPPD crystal deposition disease.

Additionally, we would like to note that a polarised light microscopic observation of a fresh wet coverslip preparation ${ }^{12}$ or a deparaffinised, unstained, coverslip preparation of a serial section, or that of histological specimens stained with safranin $\mathrm{O} /$ fast green/iron haematoxylin $^{15}$ is also useful for identification of birefringent CPPD crystals in articular tissues. 
1 Ryan LM, McCarty DJ. Calcium pyrophosphate crystal deposition disease; pseudogout; articular chondrocalcinosis. In: McCarty DJ, ed. Arthritis and allied conditions.
ed. Philadelphia: Lea and Febiger, 1989:1711-36.

2 Luna LG. Routine staining procedures. Hematoxylin and eosin stains. Manual of histologic staining methods of the Armed Forces Institute of Pathology. 3rd ed. New York: McGrawHill, 1968:32-9.

3 Tazawa K, Tsutsumi Y. Effect of prolonged staining with hematoxylin on detecting helicobacter pyroli in hematoxylin-eosin-stained gastric mucosa. Pathol Int 1998;48:448-52

4 Moskowitz RW, Harris BK, Schwartz A, Marshall G. Chronic synovitis as a manifestation of calcium crystal deposition disease. Arthritis Rheum 1971;14:109-16.

5 Gerster JC, Bund CA, Lagier R, Boussina I, Fallet GH Tendon calcifications in chondrocalcinosis. A clinical, radiologic, histologic and crystallographic study. Arthritis Rheum 1977;20:717-22

6 Lillie RD. Histopathologic technic and practical histochemistry. 3rd ed. New York: McGraw-Hill, 1965.

7 Ohira T, Ishikawa K. Hydroxyapatite deposition in articular cartilage by intra-articular injections of methylprednisolone. J Bone Joint Surg Am 1986;68:509-20.
8 Ishikawa K, Ohira T, Masuda I, Kitagawa T. Pathology and pathogenesis of crystal-induced osteoarthropathy. Journal of the Japanese Orthopaedic Association 1987;61:S11-12. 9 Ellman MH, Lavin B. Chondrocalcinosis in elderly persons. Arthritis Rheum 1975;18:43-7.

10 Utsinger PD, Zvaifler NJ, Resnick D. Calcium pyrophosphate dihydrate deposition disease without chondrocalcinosis. J Rheumatol 1975;2:258-64.

11 Resnik CS, Resnik D. Crystal deposition disease. Semin Arthritis Rheum 1983;12:390-403.

12 Masuda I, Ishikawa K. Clinical features of pseudogout attack. A survey of 50 cases. Clin Orthop 1988;229:17381.

13 Bjelle A, Crocker P, Willoughby D. Ultramicrocrystals in pyrophosphate arthropathy. Crystal identification and case report. Acta Med Scand 1980;207:89-92.

14 Ivorra J, Rosas J, Pascual E. Most calcium pyrophosphate crystals appear as non-birefringent. Ann Rheum Dis 1999; 58:582-4

15 Ishikawa K, Masuda I, Ohira T, Yokoyama M. A histological study of calcium pyrophosphate dihydrate crystaldeposition disease. J Bone Joint Surg Am 1989;71:875-86 